\title{
SYNTHESIS OF SOME NOVEL TETRAHYDRONAPHTHALENE BENZIMIDAZOLE DERIVATIVES
}

\author{
Zeynep Ates-Alagoz and Erdem Buyukbingol* \\ Department of Pharmaceutical Chemistry, University of Ankara. Faculty of Pharmacy, Ankara. Turkey.
}

\begin{abstract}
Retinoids. synthetic and natural analogues of all-trans-retinoic acid (RA), exert their biological effects with responsive elements of DNA to promote on cell differentiation and proliferation and behave as potent adipogenic hormones. Herein. we describe the synthesis of a number of novel tetrahydrotetramethylnaphthalene benzimidazole derivatives as retinoids. Analogs were prepared as depicted in Scheme 1 and 2. As is evident from both shemes. a variety of tetrahydrotetramethylnaphthalene benzimidazole derivatives have been synthesized by using an appropiate $\mathrm{NaHSO}_{3}$ addiction product
\end{abstract}

\section{Introduction}

Retinoids, natural and synthetic analogs of vitamin A (retinol) have a wide spectrum of biological activities.

Retinoic acid (RA) and retinoids play essential roles in many diverse biological events including cell diffrentiation/poliferation ${ }^{12}$ and lipid peroxidase inhibition'. These properties confer a high potential for the treatment of hyperproliferative disorders ${ }^{4}$. Clinically retinoids are used in the treatment of proliferative dermatological diseases and leukemia, and in the prevention of some tumors ${ }^{5}$. Retinoids have been shown to inhibit the growth of different tumour cell lines ${ }^{6-8}$ and to supress the induction of growth-related properties ${ }^{9}$. Therefore, retinoids have recently received considerable attention as agents that may have utility for both cancer prevention and treatment due to their cell differentiation/proliferation ${ }^{10}$. Additionally, retinoids have been shown to inhibit microsomal lipid peroxidation as effective antioxidants ${ }^{11.12}$. Recently there has been a great deal of interest in the study on the free radicals such as superoxide anion, hydroxyl anion and hydrogen peroxide which are related with atherosclerosis, nephrititis and carcinogenesis ${ }^{13.14}$. To investigate the antioxidant activity and consequently a cell differentiation and poliferation activity, some benzimidazole derivatives, related to the structure of RA and well known compound Am $580^{15}$ have been synthesized. As part of our ongoing retinoid program, we had observed that a benzimidazole-type of retinoid compound inhibits hepatic CYPIAI/2 and CYP 2BI enzymes, since these enzymes activate polycyclic hydrocarbons to their ultimate mutagenic and carcinogenic forms, and are effective in producing reactive oxygen species such as superoxide and hydroxyl radicals ${ }^{16}$. The actions of arylbenzimidazoles on the hepatic cytochrome P450 enzymes have been also reported, previously ${ }^{17}$. 


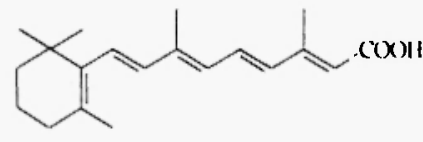

Retinoic acid

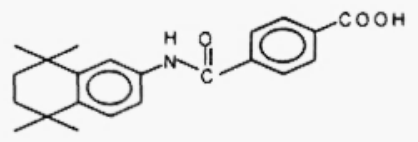

Am580

Figure 1: Structures of all-rrcmis-retinoic acid (RA) and Ain 580

\section{Experimental}

Although a number of improved synthetic routes to retinoids have been revealed in the literature. we have synthesized benzimidazole-linked tetrahydronaphthalene type compounds. The synthesis of the compounds can be divided into two parts. The first part of our synthesis. (6a-h) were carried out as shown in Scheme I. The starting material was the 2.5-dichloro-2.5-dimethyl hexane. 2 , was prepared in $56 \%$ yield by passing dry hydrogen chloride gas over 2.5-dimethyl-2.5-hexandiole. 1. as described by Boehm et all, $1994^{18}$. Toluene was alkylated by 2.5-dichloro-2.5-dimethyl hexane in dichloromethane catalyzed with aluminum chloride to produce $1,2.3,4$ tetrahydro-1.1.4.4.6-pentamethylnaphthalene. 3. in $91 \%$ yield ${ }^{18}$ followed by synthesizing of 5.6.7.8-tetrahydro5.5,8.8-tetramethyl-2-naphthalene-carboxaldehyde. 4 , was achieved by ammonium cerium nitrate $\left(\left(\mathrm{NH}_{4}\right)_{2} \mathrm{Ce}\left(\mathrm{NO}_{3}\right)_{6}\right)$ as described ${ }^{19}$. An intermediate addiction product, 5 , has been obtained by the procedure that aldehyde compound, 4 , in etanol was added to the solution $\mathrm{NaHSO}_{3}$ in water (m.p. decomp. $\left.>300^{\circ} \mathrm{C}\right)^{-0}$. To obtain the desired compounds, Weidenhagen procedure was used ${ }^{20}$. Thus, the treatment of the intermediate, 5. with substituted o-phenylenediamine afforded the expected 2-[(5.6.7,8-tetrahydro-5,5,8.8-tetramethyl-2-naphthalene)]4-or-5-substitured benzimidazole compounds ${ }^{21}, 6 \mathrm{a}-\mathrm{h}$, in several yields. The general synthesis of the compounds is seen in Scheme 1.

The second part of synthesis (Scheme 2) consists of to obtain alkyl derivatives of tetrahydrotetramethy|naphthalene-benzimidazoles at imidazole ring nitrogen. The starting material was 4-chloro-j-nitrobenzoic acid, 7, which was refluxed in methanol (or ethanol) followed by passing through the $\mathrm{HCl}$ gas to obtain 4chloro-3-nitro-methyl benzoic acid methyl/ethyl esters, 8a-b. The exchange of chloride by substituted amine, 9a-c, moiety was done according to the procedure of Goker et $\mathrm{al}^{22}$. The next step was a reduction of the nitro group. The use of $\mathrm{Zn} / \mathrm{NiCl}_{2} .6 \mathrm{H}_{2} \mathrm{O}$ in methanol gave compounds, $10 \mathrm{a}-\mathrm{c}$ in excellent yields. The condensing of these compounds with the $\mathrm{NaHSO}_{3}$ addiction product, 5 , in DMF for $70 \mathrm{hrs}$ afforded tetrahydronaphthalenebenzimidazole derivatives, 11a-b. The hydrolysis of the latter products gave final compounds, 12a-b. The physical data of the compounds are summarized in Table 1.

Melting points were determined with a Buchi SMP-20 and Buchi 9100 melting point apparatus and are uncorrected. The 'H NMR spectra were recorded with a Bruker GmbH DPX-400 (400 MHz) spectrophotometer, in DMSO- $d_{6}$ or $\mathrm{CDCl}_{3}$ unless otherwise stated, $\delta$ scale $(\mathrm{ppm})$ from internal standart TMS. The IR spectra were recorded on a Jasco FT/IR-420 spectrophotometer as potassium bromide pellets. The Mass spectra (in El mode at $70 \mathrm{eV}$ ) were recorded with a Nicromass UK. Platform II LC-MS and elemental analysis were performed on LECO 932 CHNSO. 


\section{Scheme 1}

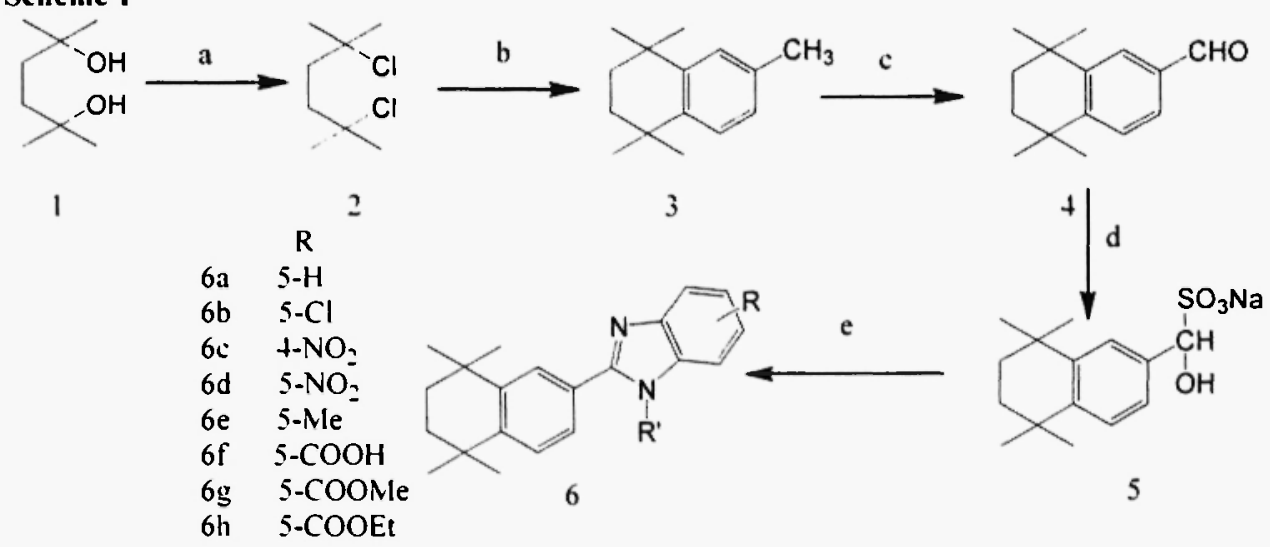
a) $\mathrm{HCl} / \mathrm{H}_{2} \mathrm{SO}_{4}, \mathrm{EtOH}$
b) Toluene, $\mathrm{AlCl}_{3}, \mathrm{CH}_{2} \mathrm{Cl}$, reflux
c) $\left(\mathrm{NH}_{4}\right)_{2} \mathrm{Ce}\left(\mathrm{NO}_{3}\right)_{6}$. AcOH, reflux
d) $\mathrm{NaHSO}_{3}$
e) Substituted o-phenelediamine. DMF, reflux

\section{Scheme 2}

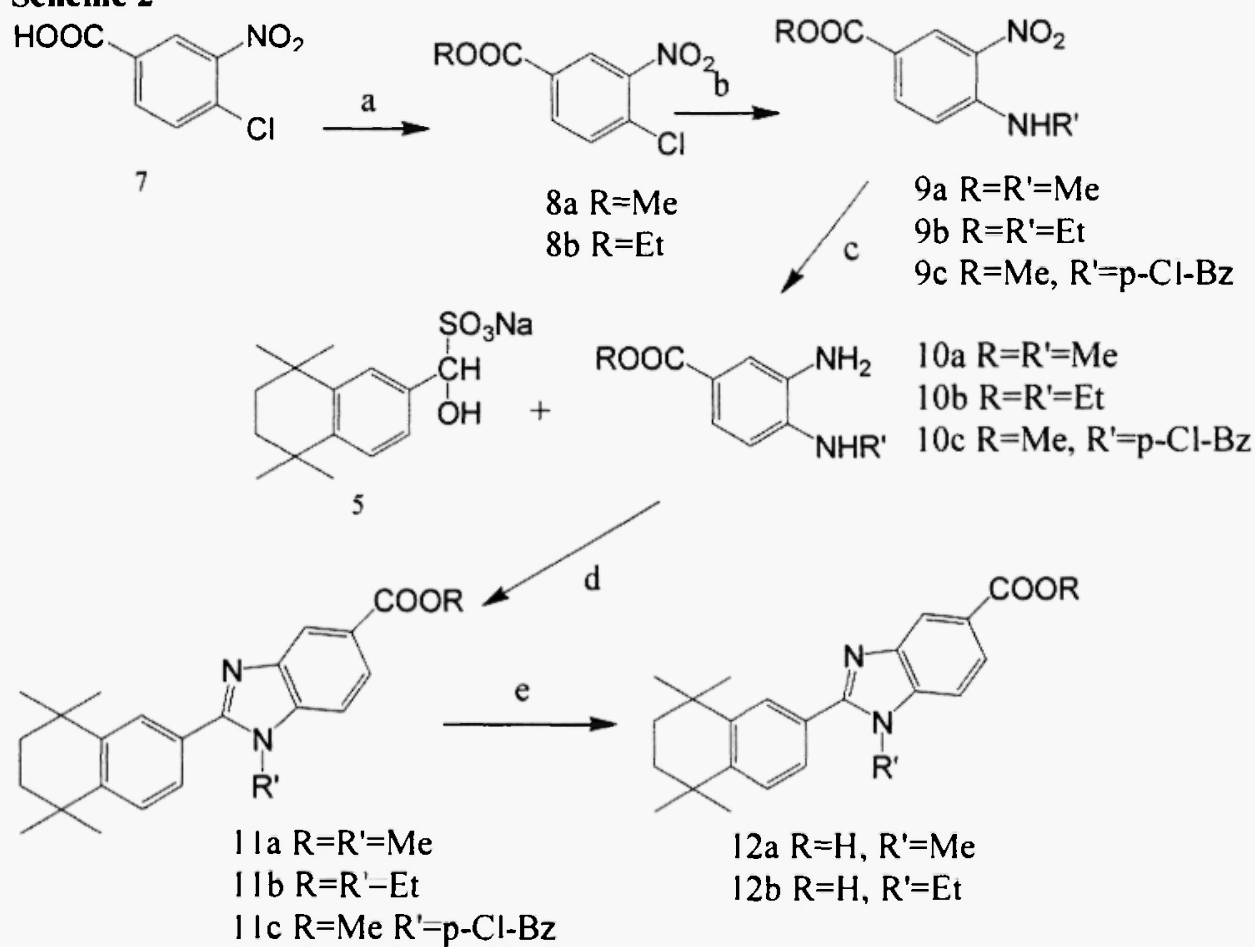

a) $\mathrm{MeOH} / \mathrm{Et}, \mathrm{HCl}$, reflux; b) $\mathrm{R}^{\prime} \mathrm{NH}_{2}$, THF, reflux; c) $\mathrm{Zn} / \mathrm{NiCl}_{2}, \mathrm{MeOH}$, reflux;

d) DMF, reflux; e) $\mathrm{NaOH}$, EtOH, reflux

Synthesis of 1,2,3,4-tetrahydro-1,1,4,4,6-pentamethylnaphthalene (3).

To a $250 \mathrm{ml}$ round bottomed flask fitted with a magnetic stirring bar and reflux condenser were added $10.0 \mathrm{~g}(54.5$ $\mathrm{mmol})$ of 2,5-Dichloro-2,5-dimethyl hexane, $10.0 \mathrm{~g}(110 \mathrm{mmol})$ of toluene and $50 \mathrm{ml}$ of dichloromethane. To this vigorously stirred solution was slowly added $100 \mathrm{mg}(0.75 \mathrm{mmol})$ of aluminum chloride which resulted in rapid evolution of gaseous hydrochloric acid. The reaction mixture was stirred at room temperature for $30 \mathrm{~min}$ followed 
by retlux for an additional $15 \mathrm{~min}$ to give a red solution containing 1.2.3.4-tetrahydro-1.1.4.4.6pentamethylnaphthalene. Alter cooling $10 \mathrm{ml}$ of $20^{\circ}$ o aqueous hydrochloric acid was added to the stirred solution and the reaction mixture turned clear/white. The organics were extracted with $2 \times 100 \mathrm{ml}$ of hexanes, washed with water and brine. dried over magnesium sulfate. filtered. concentrated and distilled to give $10.0 \mathrm{~g}(49.5 \mathrm{mmol})$ of 1.2.3.4-tetrahydro-1.1.4.4.6-pentamethylnaphthalene compound $\left(91^{\circ} \text { o yield. m.p. } 31-32^{\prime \prime C}\right)^{18}$.

Synthesis of 5,6,7,8-tetrahydro-5,5,8,8-tetrametlıyl-2-ıaphthalene-carboxaldehyde (4). This compound was synthesized as described b! Dawson et all ${ }^{14}$. To a vigorously stirred 100 " $\mathrm{C}$ mixture of $10.0 \mathrm{~g}$ (0.0494 mol) of 1.2.3.4-tetrahydro-1.1.4.4.6-pentamethylnaphthalene and $20 \mathrm{ml}$ of glacial acetic acid was added a solution of $150.0 \mathrm{~g}(0.274 \mathrm{~mol})$ of $\left(\mathrm{NH}_{4}\right)_{2} \mathrm{Ce}\left(\mathrm{NO}_{3}\right)_{6}$ in $500 \mathrm{ml}$ of $50^{\circ}$ a aqueous acetic acid over $3 \mathrm{~h}$ period. The mixture was stirred $1 / 1$, cooled. poured onto ice and extracted with petroleum ether $(3 \times 300 \mathrm{ml})$. The extract were washed 2 times with water. dried on magnesium sulphate and concentrated to give $8.5 \mathrm{~g}(78 \%)$ of compound column chromatography (silicagel. $10^{\circ}$ o Et, O/hexane) was used to obtain colourless crystals (m.p: $52-54^{\circ} \mathrm{C}$ ).

Synthesis of sulfide addiction product (5).

A procedure by Weidehagen was used ${ }^{20}$. To a solution of $6.6 \leqq(30 \mathrm{mmol})$ of 5.6.7.8-tetrahydro-5.5.8,8-tetramethyl2-naphthalene-carboxaldehy de in $20 \mathrm{ml}$ ethanol was added to the solution of $3.12 \mathrm{~g}(30 \mathrm{mmol}) \mathrm{NaHSO}_{3}$ in $20 \mathrm{ml}$ $\mathrm{H}_{2} \mathrm{O}$, stirred in ice-bath to give $7.2 \mathrm{~g}$ white precipitate which was filtered and dried. (74\% yield. m.p. decom. $>300^{\prime \prime} \mathrm{C}$ ).

General synthesis of 2-[(5,6,7,8-tetrahydro-5,5,8,8-tetramethyl-2-naphthalene)]-5- benzimidazole derivatives (6a-h).

The $\mathrm{NaHSO}_{3}$ addiction product. 5 ( $\left.10 \mathrm{mmol}\right)$, and l-substituted-3.t-diamino-benzoic acid $(10 \mathrm{mmol})$ were refluxed in DMF for 72 hours, poured onto ice-cold water with stirring. The precipitate was filtered off and extracted with ethyl acetate $(3 \times 30 \mathrm{ml})$ washed with excess amount of water followed by column chromatography for purification 21 .

Synthesis of 4-chloride,3-nitro benzoic acid methyl/ethyl ester (8a-b).

4-chloride, 3-nitro-benzoic acid ( $25 \mathrm{mmol}$ ) was refluxed in $50 \mathrm{ml}$ methanol/ethanol presence of $\mathrm{HCl}$ gas. The solvent was evaporated and $50 \mathrm{ml}$ water was added. Then neutralized with $\mathrm{NaHCO}_{3}$ to give white precipitate which was filtered and dried (m.p. and yield. respectively, $\left.8 \mathrm{a}: 63^{\circ} \mathrm{C}, 97 \% ; 8 \mathrm{~b}: 59.5^{\circ} \mathrm{C}, 95 \%\right)^{2 .}$.

Synthesis of 4-alkylamino, 3-nitro, benzoic acid methyl/ethyl ester (9a-c).

4-chloride-3-nitro-methyl/ethyl benzoat $(4.6 \mathrm{mmol})$ was refluxed in $40 \mathrm{ml}$ alkylamine (methyl amine, ethyl amine or p-Cl-Benzyl amine) and $10 \mathrm{ml}$ tetrahydrofuran for 2 hour at $40^{\circ} \mathrm{C}$. Then solvents were evaporated. water was added to give yellow precipitate which was filtered and dried (m.p. and yield. respectively, $9 \mathrm{a}: 211^{\circ} \mathrm{C} .78 \%$; $\left.9 \mathrm{~b}: 104^{0} \mathrm{C}, 84 \% ; 9 \mathrm{c}: 142^{\circ} \mathrm{C}, 89 \%\right)^{22}$.

Synthesis of 3-amino, 4-alkylamino, benzoic acid methyl/ethyl ester ( $10 \mathrm{a}-\mathrm{c})$. 4-Alkylamino-3-nitro-benzoic acid methyl/ethyl ester $(4.7 \mathrm{mmol})$ was solved with $15 \mathrm{~mL}$ methanol and reduction with $1.3 \mathrm{~g} \mathrm{NiCl}_{2} .6 \mathrm{H}_{2} \mathrm{O}$ and $1.5 \mathrm{~g} \mathrm{Zn}$. The mixture was filtered while boiling. The precipitate washed with boiled methanol and concentrated to give3-amino-4-alkyl amino-benzoic acid methyl/ethyl ester (m.p. and yield, respectively, $\left.10 \mathrm{a}: 144^{\circ} \mathrm{C}, 46 \% ; 10 \mathrm{~b}: 73^{\circ} \mathrm{C}, 34 \% ; 10 \mathrm{c}: 223^{\circ} \mathrm{C}, 96 \%\right)^{22}$.

General synthesis of I-alkyl-2-[(5,6,7,8-tetrahydro-5,5,8,8-tetramethyl-2-naphthalene)]-5- benzimidazole derivatives $(11 \mathrm{a}-\mathrm{c})$ and $(12 \mathrm{a}-\mathrm{b})$

The addiction product (5) (2mmol), and l-substituted-3-amino, 4-alkylamino-benzoic acid ( 2 mmol) were refluxed in DMF for 72 hours, poured onto ice-cold water with stirring. The precipitate was filtered off and extracted with ethyl acetate $(3 \times 30 \mathrm{ml})$ washed with excess amount of water followed by column chromatography for purification. Final products (12a-b), were obtained hydrolysis procedure from (1 la-b). Compounds $11 \mathbf{a}(0.7 \mathrm{mmol})$ or $1 \mathrm{lb}(0.7$ mmol) was solved in $1.5 \mathrm{ml}$ ethanol and were refluxed in $5 \mathrm{ml} \% 5 \mathrm{NaOH}$ for 6 hours. Reaction mixture was acidified with glasial asetic acid. The precipitate was filtered and washed with water, then crystalized from ethanol for purification to give desired compounds.

Acknowledgement: This work is partially supported by Ankara University Research Fund (97-03-00-08). TUBITAK (SBAG-1864) and ECZACIBASI Scientific Research and Award Foundation. 
Table 1. Structural and Plyysical Data for Tetrahydronahpthalene-benzimidazole compounds.

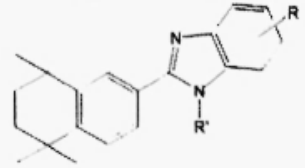

\begin{tabular}{|c|c|c|c|c|c|c|c|}
\hline Comp. & $\mathrm{R}$ & $\mathrm{R}^{\prime}$ & $\begin{array}{l}\text { M.P } \\
\text { ('C) }\end{array}$ & $\begin{array}{l}\text { Yield } \\
(\%)\end{array}$ & NMR & MASS $\mathrm{m} / \mathrm{z}$ & $\begin{array}{c}\mathrm{IR} \\
\left(\mathrm{KBr} \mathrm{cm}^{\prime}\right)\end{array}$ \\
\hline $6 a$ & 11 & H & 249 & 19 & 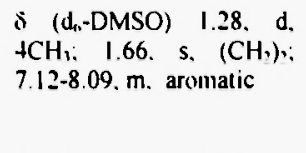 & $\begin{array}{l}30+\left(\mathrm{M}^{+}\right), 305(\mathrm{M}+\mathrm{l}), 290((\mathrm{M}+1)- \\
\left.\mathrm{CH}_{3}\right), 289\left(\mathrm{M}-\mathrm{CH}_{3}\right), 275((\mathrm{M}+1)- \\
\left.2 \mathrm{CH}_{3}\right), 274\left(\mathrm{M}-2 \mathrm{CH}_{3}\right), 260((\mathrm{M}+1)- \\
\left.3 \mathrm{CH}_{3}\right), 259\left(\mathrm{M}-3 \mathrm{CH}_{2}\right), 245((\mathrm{M}+1)- \\
\left.\mathrm{fCH}_{2}\right)\end{array}$ & $\begin{array}{ll}3050- & \mathrm{CH}_{2} . \\
\mathrm{CH}, & 2750- \\
=\mathrm{CH}\end{array}$ \\
\hline $6 b$ & $5-\mathrm{Cl}$ & $\mathrm{H}$ & $? 18$ & 16 & $\begin{array}{l}\delta\left(\mathrm{d}_{1}-\mathrm{DMSO}\right) \quad 1.31 . \mathrm{d} . \\
+\mathrm{CH}: 1.68 \text {. s. }\left(\mathrm{CH}_{3}\right), \\
7.19-8.13 . \text { m. aromatic }\end{array}$ & $\begin{array}{l}338\left(\mathrm{M}^{+}\right) \cdot 340 \quad(\mathrm{M}+2) .341 \\
(\mathrm{M}+3) .342(\mathrm{M}+4) \cdot 323\left(\mathrm{M}-\mathrm{CH}_{3}\right) . \\
325((\mathrm{M}+2)-\mathrm{CH}) .308\left(\mathrm{M}-2 \mathrm{CH}_{3}\right) \\
293\left(\mathrm{M}-3 \mathrm{CH}_{3}\right)\end{array}$ & $\begin{array}{l}2959-2859 \\
\mathrm{CH}:-\mathrm{CH}:\end{array}$ \\
\hline $6 c$ & $4-\mathrm{NO}$ & $\mathrm{H}$ & 198 & 30 & $\begin{array}{l}\delta\left(\mathrm{CDCl}_{1}\right) 1.37 . \mathrm{s} .2 \mathrm{CH}: \\
1.43 . \mathrm{s}, \quad 2 \mathrm{CH}_{3}: 1.78 . \mathrm{s} \\
(\mathrm{CH}), \quad 7.6-8.3 . \mathrm{m} . \\
\text { aromatic) }\end{array}$ & $\begin{array}{l}350(\mathrm{M}+1), 335\left((\mathrm{M}+1)-\mathrm{CH}_{3}\right), 319 \\
\left(\left(\mathrm{M}-2 \mathrm{CH}_{3}\right) .305\left(\mathrm{M}-3 \mathrm{CH}_{3}\right) \cdot 289(\mathrm{M}-\right. \\
\left.4 \mathrm{CH}_{3}\right), 289\left(335-\mathrm{NO}_{3}\right)\end{array}$ & $\begin{array}{ll}1541 . & 1362 \\
\left(\mathrm{NO}_{2}\right) & \end{array}$ \\
\hline $6 d$ & 5-NO, & $\mathrm{H}$ & 290 & 31 & $\begin{array}{l}\delta\left(\mathrm{CDCl}_{i}\right) 1.31 . \mathrm{s} .2 \mathrm{CH}_{1} \\
1.33 . \mathrm{s} . \quad 2 \mathrm{CH}_{\mathrm{i}}: 1.72 . \mathrm{s} . \\
\left(\mathrm{CH}_{2}\right): \quad 7 .+3-8.51 . \quad \mathrm{m} . \\
\text { aromatic }\end{array}$ & 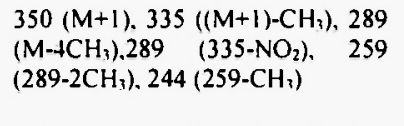 & $\begin{array}{ll}1537 . & 1344 \\
\left(\mathrm{NO}_{21}\right. & \end{array}$ \\
\hline $6 e$ & 5-Me & $\mathrm{H}$ & 222 & 37.5 & $\begin{array}{l}\delta\left(\mathrm{CDCl}_{3}\right) 1.04 . \mathrm{s} .2 \mathrm{CH}_{1}: \\
1.24 . \mathrm{s} .2 \mathrm{CH}_{1} ; 1.62 . \mathrm{dd} . \\
(\mathrm{CH},)_{2}: 2+1 . \mathrm{s} .-\mathrm{CH}_{3} ; 7.04- \\
8.3, \mathrm{~m} \text {. aromatic }\end{array}$ & $\begin{array}{l}319(\mathrm{M}+1), 318\left(\mathrm{M}^{+}\right), 303(\mathrm{M}- \\
\left.\mathrm{CH}_{3}\right), 289\left((\mathrm{M}+1)-2 \mathrm{CH}_{3}\right), 288(\mathrm{M}- \\
\left.2 \mathrm{CH}_{3}\right), 273\left(\mathrm{M}-3 \mathrm{CH}_{3}\right), 259((\mathrm{M}+1)- \\
4 \mathrm{CH} 3)\end{array}$ & $\begin{array}{l}3050-2850 \\
\mathrm{CH}_{3} \\
\mathrm{CH}_{2}=\mathrm{CH}\end{array}$ \\
\hline $6 f$ & $5-\mathrm{COOH}$ & $\mathrm{H}$ & 215 & 34 & $\begin{array}{l}\delta \quad\left(\mathrm{d}_{4}-\mathrm{DMSO}\right) \quad 1.33, \mathrm{~d} . \\
+\mathrm{CH}_{3}, \mathrm{I.70}, \mathrm{s},\left(\mathrm{CH}_{2}\right)_{2}: 7.5- \\
8.3 . \mathrm{m} . \quad \text { aromatic, } 13.00 . \\
\text { broad s. }-\mathrm{OH}\end{array}$ & $\begin{array}{l}348\left(\mathrm{M}^{+}\right), 349(\mathrm{M}+1), 303(\mathrm{M}- \\
\mathrm{COOH}), 333\left(\mathrm{M}-\mathrm{CH}_{3}\right), 318(\mathrm{M}- \\
2\left(\mathrm{CH}_{3}\right), 303\left(\mathrm{M}-\left(\mathrm{CH}_{3}\right)\right.\end{array}$ & $\begin{array}{l}1684 \\
\mathrm{COOH}\end{array}$ \\
\hline $6 \mathrm{~g}$ & 5-COOMe & $\mathrm{H}$ & 223 & 30.2 & $\begin{array}{l}\delta\left(\mathrm{CDCl}_{3}\right) 0.96, \mathrm{~s}, 2 \mathrm{CH}_{3} ; \\
1.22, \mathrm{~s}, 2 \mathrm{CH}_{3}, 1.59, \mathrm{dd}, \mathrm{J} 8 \\
\mathrm{~Hz}\left(\mathrm{CH}_{2}\right)_{2} ; 3.88, \mathrm{~s},-\mathrm{OCH}_{3} ; \\
7.31-8.34, \mathrm{~m} \text {, aromatic }\end{array}$ & $\begin{array}{lll}362\left(\mathrm{M}^{+}\right), 363(\mathrm{M}+1), 347 & (\mathrm{M}- \\
\left.\mathrm{CH}_{1}\right), 332\left(\mathrm{M}-2 \mathrm{CH}_{2}\right), 331 & (\mathrm{M}- \\
\left.\mathrm{OCH}_{3}\right), 317\left(\mathrm{M}-3 \mathrm{CH}_{3}\right), & 304 \\
\left((\mathrm{M}+1)-\mathrm{COOCH}_{3}\right), 303 & (\mathrm{M}- \\
\left.\mathrm{COOCH}_{3}\right), 303\left((\mathrm{M}+1)-4 \mathrm{CH}_{1}\right) & \end{array}$ & $\begin{array}{l}1693 \\
\text { COOMe }\end{array}$ \\
\hline $6 \mathrm{~h}$ & 5-COOEt & $\mathrm{H}$ & 129 & 84 & $\begin{array}{l}\delta \quad\left(\mathrm{d}_{6}-\mathrm{DMSO}\right) \quad 133, \mathrm{~d}, \\
4 \mathrm{CH}_{3} ; 1.36, \mathrm{t},-\mathrm{CH}_{2} \mathrm{CH}_{3} ; \\
1.7, \mathrm{~s},\left(\mathrm{CH}_{2}\right)_{2} ; 4.35, \mathrm{q},- \\
\mathrm{OCH}_{2}-\mathrm{CH}_{3} ; 7.5=8.2, \mathrm{~m} . \\
\text { aromatic }\end{array}$ & $\begin{array}{l}377(\mathrm{M}+\mathrm{l}), 362\left((\mathrm{M}+1)-\mathrm{CH}_{3}\right), 348 \\
\left((\mathrm{M}+1)-\mathrm{CH}_{2} \mathrm{CH}_{3}\right), \quad 332((\mathrm{M}+1)- \\
\left.3 \mathrm{CH}_{1}\right), 332\left((\mathrm{M}+1)-\mathrm{OCH}_{2} \mathrm{CH}_{3}\right), 320 \\
(348-\mathrm{C} 0), \quad 304 \quad((\mathrm{M}+1)- \\
\left.\mathrm{COOCH}_{2} \mathrm{CH}_{3}\right)\end{array}$ & $\begin{array}{l}1715 \\
\text { COOEt }\end{array}$ \\
\hline I la & 5-COOMe & $\mathrm{Me}$ & 250 & 51.5 & $\begin{array}{l}\delta\left(\mathrm{CDCl}_{3}\right) 1.36, \mathrm{~d}, 4 \mathrm{CH}_{3} \\
1.76, \mathrm{~s},\left(\mathrm{CH}_{2}\right)_{2} ; 3.00, \mathrm{~d}, \mathrm{~N}- \\
\mathrm{CH}_{13}, 3.89, \mathrm{~s},-\mathrm{OCH}_{3}, 6.42- \\
8.22, \mathrm{~m} \text {, aromatic }\end{array}$ & $\begin{array}{l}377(\mathrm{M}+1), 376\left(\mathrm{M}^{+}\right), 362((\mathrm{M}+1)- \\
\left.\mathrm{CH}_{3}\right), 361\left(\mathrm{M}-\mathrm{CH}_{3}\right), 347((\mathrm{M}+1)- \\
\left.2 \mathrm{CH}_{3}\right), 346\left(\mathrm{M}-2 \mathrm{CH}_{3}\right), 346((\mathrm{M}+1)- \\
\left.\mathrm{OCH}_{3}\right), 345 \quad\left(\mathrm{M}-\mathrm{OCH}_{3}\right), 332 \\
\left((\mathrm{M}+\mathrm{l})-3 \mathrm{CH}_{1}\right), 331\left(\mathrm{M}-3 \mathrm{CH}_{1}\right), 317 \\
\left(\mathrm{M}-\mathrm{COOCH}_{3}\right), 317\left((\mathrm{M}+1)-4 \mathrm{CH}_{3}\right)\end{array}$ & $\begin{array}{l}1628 \\
\text { COOMe }\end{array}$ \\
\hline $11 \mathrm{~b}$ & $5-\mathrm{COOEt}$ & Et & 178 & 16.8 & $\begin{array}{l}\delta\left(\mathrm{CDCH}_{3}\right) \mathrm{I} .36, \mathrm{~d}, 4 \mathrm{CH}_{3} \\
\mathrm{I} .45, \mathrm{t}, \mathrm{N}-\mathrm{CH}_{2} \mathrm{CH}_{3} ; 1.54, \mathrm{t}, \\
\mathrm{O}-\mathrm{CH}_{2} \mathrm{CH}_{3} ; \mathrm{I} .7, \mathrm{~s},\left(\mathrm{CH}_{2}\right)_{2 ;} \\
4.3, \mathrm{q}, \mathrm{N}-\mathrm{CH}_{2} \mathrm{CH}_{3} ; 4.4, \mathrm{q}, \\
\mathrm{O}-\mathrm{CH}_{2} \mathrm{CH}_{3} ; 7.28-8.57, \mathrm{~m}, \\
\text { aromatic }\end{array}$ & $\begin{array}{l}405(\mathrm{M}+1), 404\left(\mathrm{M}^{4}\right), 390((\mathrm{M}+1)- \\
\left.\mathrm{CH}_{3}\right), 389\left(\mathrm{M}-\mathrm{CH}_{1}\right), 374\left(\mathrm{M}-2 \mathrm{CH} \mathrm{CH}_{1}\right), \\
360\left((\mathrm{M}+1)-3 \mathrm{CH}_{1}\right), 360 \quad(\mathrm{M}- \\
\left.\mathrm{OCH}_{2} \mathrm{CH}_{3}\right), 359\left(\mathrm{M}-3 \mathrm{CH}_{3}\right), 344(\mathrm{M}- \\
\left.4 \mathrm{CH}_{1}\right), 333\left((\mathrm{M}+1)-\mathrm{COOCH}_{2} \mathrm{CH}_{3}\right)\end{array}$ & $\begin{array}{l}1710 \\
\text { COOEt }\end{array}$ \\
\hline $11 \mathrm{c}$ & 5.COOMe & $\mathrm{p}-\mathrm{Cl}-\mathrm{B} z$ & 209 & 16.8 & $\begin{array}{l}\delta\left(\mathrm{CDCl}_{1}\right) \mathrm{I} .08, \mathrm{~s}, 2 \mathrm{CH}_{3} \\
1.23, \mathrm{~s}, 2 \mathrm{CH}_{3} ; 1.62, \mathrm{~d}, \\
\left(\mathrm{CH}_{2}\right)_{2} ; 3.88, \mathrm{~s},-\mathrm{OCH}_{3} \\
5.37, \mathrm{~s}, \mathrm{~N}-\mathrm{CH}_{2} ; 7.1 \mathrm{I}-8.51 \\
\text { m, aromatic }\end{array}$ & $\begin{array}{l}488(\mathrm{M}+2), 486\left(\mathrm{M}^{*}\right), 471(\mathrm{M}- \\
\left.\mathrm{CH}_{1}\right), 455\left((\mathrm{M}+1)-2 \mathrm{CH}_{3}\right), 455(\mathrm{M}- \\
\left.\mathrm{OCH}_{3}\right), 429\left((\mathrm{M}+2)-\mathrm{COOCH}_{3}\right)\end{array}$ & $\begin{array}{l}1708 \\
\text { COOMe }\end{array}$ \\
\hline $12 a$ & $5-\mathrm{COOH}$ & $\mathrm{Me}$ & 282 & 41.5 & $\begin{array}{l}\delta\left(d_{1}-D M S O\right) \quad 1.33, d, \\
4 \mathrm{CH}_{1} ; 1.72, \mathrm{~s},\left(\mathrm{CH}_{2}\right)_{2} ; 3.9, \\
\mathrm{~s}, \mathrm{~N}-\mathrm{CH}_{1}, 7.5-8.3, \mathrm{~m}, \\
\text { aromatic }\end{array}$ & $\begin{array}{l}363(\mathrm{M}+1), 348\left((\mathrm{M}+1)-\mathrm{CH}_{3}\right), 346 \\
\left.((\mathrm{M}+\mathrm{I})-\mathrm{OH}), 332(\mathrm{M})-2 \mathrm{CH}_{1}\right), 318 \\
(346-\mathrm{CO}), 318\left((\mathrm{M}+1)-3 \mathrm{CH}_{3}\right), 318 \\
((\mathrm{M}+\mathrm{I})-\mathrm{COOI}), 303\left((\mathrm{M}+1)-4 \mathrm{CH}_{3}\right)\end{array}$ & $\begin{array}{l}1664 \\
\mathrm{COOH}\end{array}$ \\
\hline $12 b$ & $5 \cdot \mathrm{COOH}$ & $\mathrm{Et}$ & 286 & 41.5 & $\begin{array}{l}\delta\left(\mathrm{CDCl}_{3}\right) 1.27, \mathrm{~d}, 4 \mathrm{CH}_{3} ; \\
\mathrm{I} .46, \mathrm{I},\left(\mathrm{CH}_{2} \mathrm{CH}_{1}\right), 1.67, \mathrm{~s} \text {, } \\
\left(\mathrm{CH}_{2}\right)_{2} ; 4.24, \mathrm{q},\left(\mathrm{CH}_{2} \mathrm{CH}_{3}\right) ; \\
7.39-8.56, \mathrm{~m} \text {. aromatic }\end{array}$ & $\begin{array}{l}377(\mathrm{M}+\mathrm{I}), 376\left(\mathrm{M}^{*}\right), \quad 361(\mathrm{M}) \cdot- \\
\left.\left.\mathrm{CH}_{1}\right), 346(\mathrm{M})-2 \mathrm{CH}_{3}\right), 331(\mathrm{M})- \\
\left.4 \mathrm{CH}_{3}\right), 317\left(\mathrm{M}-\mathrm{COOCH}_{3}\right)\end{array}$ & $\begin{array}{l}1671 \\
\mathrm{COOH}\end{array}$ \\
\hline
\end{tabular}




\section{References}

I. I. Safonova. C. Darimont. E. Amri. P. Grimaldi. G. Ailhaud. U. Reichert. B. Slıroot : Moleculur and Cellular Endocrinolog:. 104, 201 (1994).

2. M.B. Sporn. A.B. Roberts: Cancer Res., 43, 3034 (1993).

3. M. Hiramatsu. L. Packer: .Methods Enzzmol.. 190, 273 (1990).

4. B.A. Pawson. C.W. Ehmann. M.I. Sherman: J. Med. Chem.. 25, 1269 (1982).

5. W. Bollag. E. Holdener: Ann. Oncology. 3, 513 (1992).

6. M.B. Sporn. A.B. Roberts. D.S. Goodman, in: The retinoids. 1. Academic press. Orlando 1984.

7. P.P. Lee. M.T. Lee. K.M. Darcy. K. Shudo: Ip M. M.: Endocrinol., 136, 1707 (1995).

8. M. Reiss, C. Camba-Vitalo. A.C. Sartorelli: Cancer Treat. Res., 70, 201 (1986).

9. R. Lotan. G. Neumann. D. Lotan: Cancer Res., 40, 1097 (1980).

10. M.S. Tallman. P.H. Wiernik: J. Clin. Pharmacol.. 32, 868 (1992).

11. V.M. Samokyszyn. L.J. Marnett: Methods in Enzymol, 190, 281 (1990).

12. G. Levin. S. Mokady: Free Rud. Biol. Med., 17, 77 (1994).

13. C.A. Rice-Evans. A.T. Diplock: Techniques in Free Radical Research. Elsevier, Amsterdam, 1991.

14. H. Kappus: Chem. Physic. Lipids, 45, 105 (1987).

15. T. Oikawa. I. Okavasu, H. Ashino, I. Morita, S. Murota, K. Shudo: Eur. J. Pharmacol., 249, 113 (1993).

16. Z. Ates. S. Suzen, E. Buyukbingol. B. Can-Eke, M. Iscan: Farmaco 52, 703 (1997).

17. M.Y. Iscan. E. Buyukbingol, M. Iscan, F. Sahin, C. Safak: Comp. Biochem. Physiol., 92C, 109 (1989).

18. M.F. Boehm. L. Zhang, B.A. Badea, S.K. White, D.E. Mais, E. Merger, C.M. Suto, M.E. Goldman, R.A. Heyman: J. Med. Chem., 37, 2930 (1994).

19. M.I. Dawson, P.D. Hobbs, K.A. Derdzinski, W. -W. Chao, G. Frenking, G.H. Loew, A.M. Jetten, J.L. Napoli, J.B. Williams, B.P. Sani, Jr. J.J. Wille, L.J. Schiff: J.Med. Chem., 32, 1504 (1989).

20. R. Weidenhagen: Ber, 69, 2263 (1966).

21. H.F. Ribely, G.W. Ftickett, G.M. Timmis: J. Het. Chem., 2, 453 (1965).

22. H. Goker, E. Tebrizli, U. Abbasoglu: Farmaco, 51, 53 (1966).

\section{Received on July 11, 2001}

